\section{BMJ Open Respiratory Research}

\title{
Characterisation of ICU sleep by a commercially available activity tracker and its agreement with patient- perceived sleep quality
}

\author{
Patricia R Louzon (D) , Jessica L Andrews, ${ }^{2}$ Xavier Torres, ${ }^{3}$ Eric C Pyles, ${ }^{1}$ \\ Mahmood H Ali, ${ }^{4}$ Yuan Du, ${ }^{5}$ John W Devlin ${ }^{6,7}$
}

\section{ABSTRACT}

Background A low-cost, quantitative method to evaluate sleep in the intensive care unit (ICU) that is both feasible for routine clinical practice and reliable does not yet exist. We characterised nocturnal ICU sleep using a commercially available activity tracker and evaluated agreement between tracker-derived sleep data and patient-perceived sleep quality.

Patients and methods A prospective cohort study was performed in a 40-bed ICU at a community teaching hospital. An activity tracker (Fitbit Charge 2) was applied for up to 7 ICU days in English-speaking adults with an anticipated ICU stay $\geq 2$ days and without mechanical ventilation, sleep apnoea, delirium, continuous sedation, contact isolation or recent anaesthesia. The RichardsCampbell Sleep Questionnaire (RCSQ) was administered each morning by a trained investigator.

Results Available activity tracker-derived data for each ICU study night (20:00-09:00) (total sleep time (TST), number of awakenings (\#AW), and time spent light sleep, deep sleep and rapid eye movement (REM) sleep) were downloaded and analysed. Across the 232 evaluated nights (76 patients), TST and RCSQ data were available for 232 (100\%), \#AW data for 180 (78\%) and sleep stage data for 73 (31\%). Agreement between TST (349 $168 \mathrm{~min})$ and $\mathrm{RCSQ}$ Score was moderate and significant $(\mathrm{r}=0.34 ; 95 \% \mathrm{Cl}$ 0.18 to 0.48). Agreement between \#AW (median (IQR), 4 (2-9)) and RCSQ Score was negative and non-significant ( $r=-0.01 ; 95 \% \mathrm{Cl}-0.19$ to 0.14$)$. Agreement between time (min) spent in light (259 (182 to 328)), deep (43 \pm 29$)$, and REM (47 (28-72)) sleep and RCSQ Score was moderate but non-significant (light $(r=0.44,95 \% \mathrm{Cl}-0.05$ to 0.36 ); deep sleep $(r=0.44,95 \% \mathrm{Cl}-0.11$ to 0.15$)$ and REM sleep $(r=0.44 ; 95 \% \mathrm{Cl}-0.21$ to 0.21$)$ ).

Conclusions A Fitbit Charge 2 when applied to nonintubated adults in an ICU consistently collects TST data but not \#AW or sleep stage data at night. The TST moderately correlates with patient-perceived sleep quality; a correlation between either \#AW or sleep stages and sleep quality was not found.

\section{INTRODUCTION}

Patients often complain of poor sleep during their intensive care unit (ICU) admission. ${ }^{1}$ Disrupted sleep in this setting may increase

\section{Key Messages}

Can a commercially available activity tracker routinely report nocturnal sleep data in the intensive care unit (ICU) and do these assessments agree with patient-perceived sleep quality?

- Use of a Fitbit Charge 2 in the ICU consistently collects total sleep time (TST) but not number of awakenings (\#AW) or sleep stage data.

- Fitbit Charge 2-derived TST moderately correlates with patient-perceived sleep quality; a correlation between either \#AW or sleep stage and sleep quality was not found.

delirium risk and prolong mechanical ventilation. ${ }^{2}$ The use of sleep improvement protocols in the ICU, while being shown to reduce noise and patient interruption ${ }^{3}$ and lower delirium, ${ }^{4}$ have generally not been shown to improve sleep quality. ${ }^{1}$ An important barrier to demonstrating ICU sleep protocols improve sleep quality is the current lack of a low-cost, quantitative method to evaluate sleep in the ICU that is both reliable and feasible to use in daily practice.

While subjective, patient-derived sleep evaluations like the Richards Campbell Sleep Questionnaire (RCSQ) may help patients communicate their perceived sleep quality to clinicians, these instruments are challenging to use in patients who are sedated or who have delirium. ${ }^{5}$ While polysomnography (PSG) remains the gold standard method to measure sleep, in the ICU, PSG results can be difficult to score with traditional PSG scoring criteria given the relative absence of $\mathrm{K}$ complexes and sleep spindles in the critically ill and the frequent use of medications known to disrupt sleep architecture and regulation. ${ }^{67}$ Moreover, PSG is not a feasible method by which to routinely evaluate sleep in the ICU. ${ }^{1}$ Research-grade actigraphs have 
been shown to be feasible for use in the ICU but have not been shown to consistently agree with PSG assessments in critically ill adults. Moreover, these actigraphs are expensive and generally not available in most ICUs. ${ }^{89}$

Commercial activity trackers are readily available, have a relatively low cost, and if applied to critically ill adults would be unlikely to interfere with ICU daily care. These trackers have shown to quantitatively characterise sleep in healthy patients as well as actigraphy ${ }^{10} 11$ or PSG. ${ }^{12-14}$ However, published reports of their use in the ICU do not exist. Characterising the ability of an activity tracker to generate sleep quality data in the ICU, and how these data compare to patient-perceived sleep quality, are important first steps when determining the potential role of activity trackers in this setting.

The primary objective of this study was to evaluate the ability of a commercially available activity tracker to generate nocturnal values for total sleep time (TST), sleep awakenings (\#AW), time spent at light, deep and REM sleep in adult, non-intubated ICU patients. A secondary objective of this study was to measure agreement between the tracker-derived TST, \#AW and sleep state values and patient-perceived sleep quality. Based on published commercial activity tracker research in healthy adults we hypothesised that TST, \#AW and sleep state values could be consistently collected in critically ill adults and each of these values would significantly positively correlate with patient-perceived sleep quality.

\section{METHODS}

\section{Setting}

This prospective, single-centre, cohort study was conducted in the 40-bed medical/cardiac ICU in a 1350-bed community teaching hospital (Advent Health; Orlando, Florida, USA). AdventHealth Institutional review board approval (study number 1220470-9) and informed consent were obtained prior to study enrolment. All patients were managed with existing ICU pain assessment and treatment, spontaneous breathing and early mobilisation protocols. Daytime napping is discouraged. While earplugs and eye masks were available in the unit for clinicians to employ at their clinical discretion, the use of these or other sleep improvement strategies were not protocolised during the study period.

\section{Patients}

On each weekday, all adults ( $\geq 18$ years) admitted to the study ICU underwent a preliminary study screen by a clinical pharmacist. A study investigator then screened and removed patients from further consideration who had one or more of the following factors that could affect the ability of the activity tracker to evaluate sleep and/or the patient to self-report sleep quality using the RCSQ: paraplegia/quadriplegia, history of sleep apnoea/insomnia, anaesthesia within 24 hours, mechanical obstruction to tracker placement, delirium, contact isolation, nonEnglish speaking, blind or deaf, or who were currently receiving continuous sedatives. Patients meeting all study criteria were administered the Epworth Sleepiness Scale (ESS) and excluded if the ESS was $>16$ in an effort to remove patients from the study with an undiagnosed or undocumented pre-existing sleep condition that could confound TST measurements. ${ }^{15}$

\section{Patient and public involvement}

Patient complaints of poor sleep in the ICU and persistence after ICU discharge are well established. ${ }^{16}{ }^{17}$ Increasingly, healthy adults are measuring their sleep at home using a commercially available activity tracking device. ${ }^{18}$ ICU clinicians report the current lack of a lowcost, easy-to-use, quantitative method to evaluate sleep in the ICU as an unmet need. ${ }^{19}$ Patients were not directly involved in the protocol development nor involved in study design, recruitment or the conduct of the study. Results will not be disseminated directly to study participants; study participants were notified of this limitation in the consent process. The burden of the intervention was not assessed by patients.

\section{Interventions}

The Fitbit Charge 2 (Fitbit; San Francisco, California, USA $)^{18}$ was chosen as the commercially available activity tracker for the study given its low cost, its high global market share at the time of study and its extensive investigation as a sleep monitoring device in healthy adults. ${ }^{20}{ }^{21}$ The RCSQ was chosen to evaluate patient-perceived sleep quality in the study given it has been validated against PSG in the ICU. ${ }^{5}$

\section{Assessments}

The Fitbit Charge 2 was applied to each patient's preferred wrist over the radial tip by a study investigator to be worn continuously until ICU discharge, 7 days elapsed or a study exclusion criterion occurred. Temporary removal during procedures was permitted. Each morning, a study investigator, after confirming proper tracker placement and full battery charge, synched the Fitbit with its smartphone-enabled application using a Bluetooth connection and downloaded sleep data from the prior nocturnal (20:00 to 09:00) period.

The Fitbit Charge 2 was compared with the RCSQ. Neither actigraphy nor PSG was deemed necessary to incorporate in our study given the results of prior healthyadult validation studies showing agreement between the Fitbit Charge 2 and both actigraphy and PSG. ${ }^{10-14}$ Patientperceived sleep quality was measured daily between 08:00 and 13:00 using the RCSQ completed by the patient under supervision of a trained investigator. ${ }^{5}$ Responses for each domain were recorded on a 100-mm Visual Analogue Scale and averaged across the five domains to generate an overall RCSQ Score. To better characterise sleep quality distribution, <33 was deemed to denote poor sleep, 33-66 fair sleep and $>66$ good sleep. ${ }^{22}$ 


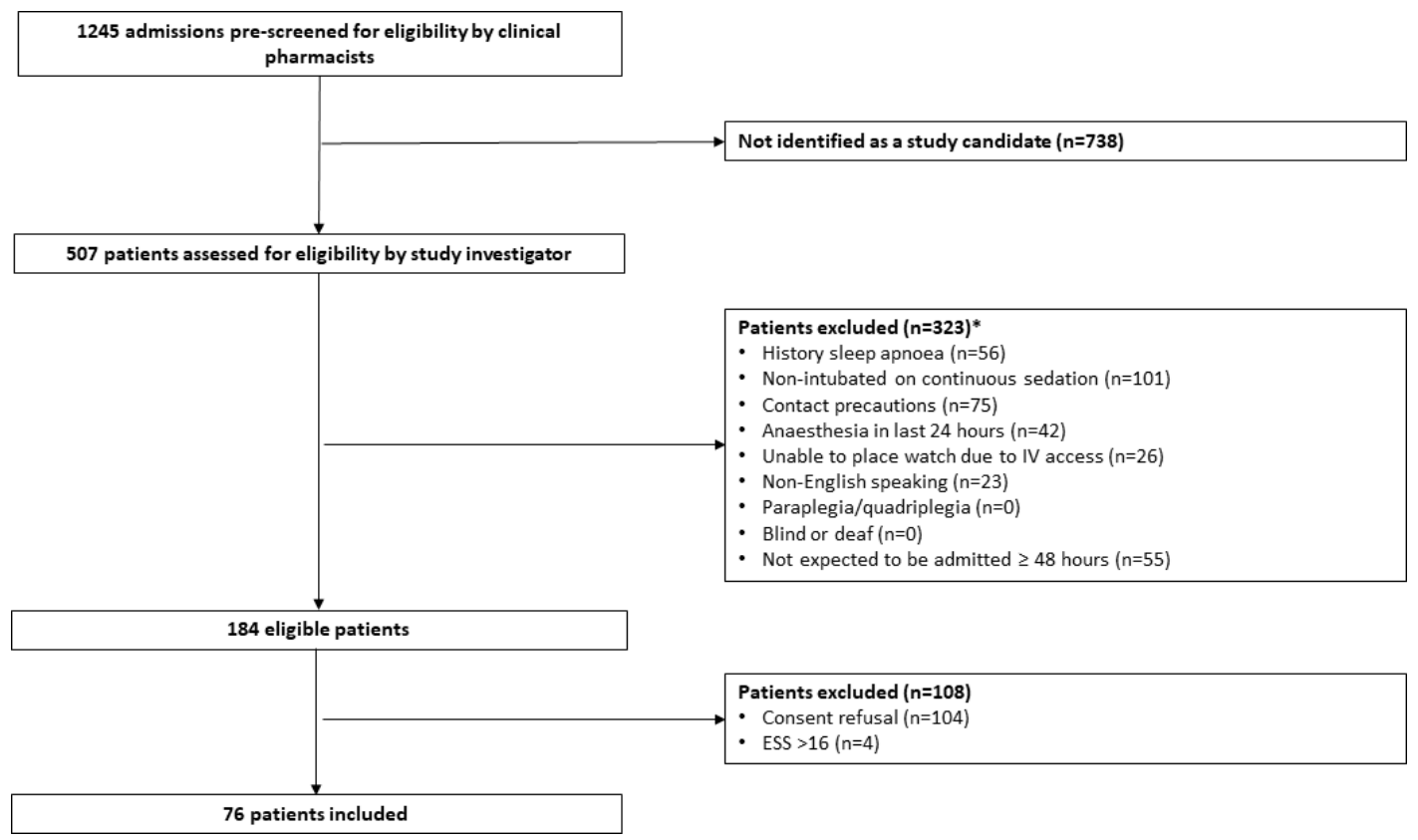

Figure 1 Patient screening and enrolment. Some patients had more than one exclusion criteria. ESS, Epworth Sleepiness Scale; IV, intravenous.

\section{Statistical analysis}

When extreme skewness was detected for any of the Fitbit outcomes, values were transformed to approximately normal distribution. Therefore, the \#AW were costransformed, light sleep time was log-transformed and REM sleep was square root-transformed. Each outcome was then evaluated for correlation with the RCSQ Score using mixed, repeated measures models that incorporated correlation structure on random effects and residuals. ${ }^{23}$ CIs were estimated using a 1000 times cluster bootstrap method. All analysis was performed using SAS V.9.4 (SAS Institute, Cary, North Carolina, USA).

\section{RESULTS}

Among 507 patients evaluated, 76 (15\%) were enrolled (age $57 \pm 15$ years; $62 \%$ male, ICU length of stay (9 (4-15) days) between December 2017 and June 2019 (figure 1). The vast majority of patients were enrolled within 2 days of ICU admission and most were admitted to the ICU because of an acute on chronic cardiac/pulmonary condition (eg, decompensated heart failure and/or pulmonary hypertension). For these 76 patients, the Fitbit was applied and an RCSQ was completed for 232 nights $/$ days (median $(\mathrm{IQR})$ average $=2(1-5)$ ). Among the 232 nights, RCSQ and TST data were available for $232(100 \%)$, \#AW for 180 (78\%) and sleep stages for 73 $(31 \%)$. Ten (13\%) patients had no nights with \#AW data; 27 (36\%) had missing data for $\geq 1$ night. Thirty (39\%) patients had no nights with sleep stage data; 32 (42\%) had missing data for $\geq 1$ night.

Agreement between each sleep measure and the RCSQ Score (on nights where both available) are shown in the table 1 . Only TST showed significant agreement with the RCSQ Score. ${ }^{24}$ No patients had a sustained heart rate $\geq 100$ beats per minute during any nocturnal period. The RCSQ Scores (mean, 52 255 ) were evenly distributed among poor $(30 \%)$, fair $(35 \%)$ and good $(35 \%)$ sleep.

Table 1 Activity tracker sleep outcomes and agreement with patient-perceived sleep quality

\begin{tabular}{lllrr}
\hline Activity tracker sleep outcome & $\begin{array}{l}\text { Nights data available } \\
\text { (number (\%)) }\end{array}$ & $\begin{array}{l}\text { Average among } \\
\text { nights with data }\end{array}$ & $\begin{array}{l}\text { Agreement with } \\
\text { RCSQ result† }\end{array}$ & \multicolumn{1}{c}{ 95\% Cl } \\
\hline Total sleep time (min) & $232(100)$ & $349 \pm 168$ & 0.34 & 0.18 to 0.48 \\
\hline Nocturnal awakenings (number) & $180(78)$ & $4(2$ to 9$)$ & -0.01 & -0.19 to 0.14 \\
\hline Light sleep (min) & $73(31)$ & $259(182$ to 328) & 0.44 & -0.05 to 0.36 \\
\hline Deep sleep (min) & $73(31)$ & $43 \pm 29$ & 0.44 & -0.11 to 0.15 \\
\hline REM sleep (min) & $73(31)$ & $47(28$ to 72$)$ & 0.44 & -0.21 to 0.21 \\
\hline
\end{tabular}

*Based on the 232 ICU nights patients were evaluated.

$\dagger$ Agreement presented as $r$ (correlation coefficient).

$\ddagger$ Results presented as either mean \pm SD or median IQR.

ICU, intensive care unit; RCSQ, Richards Campbell Sleep Questionnaire; REM, rapid eye movement. 


\section{DISCUSSION}

Our study represents the first published investigation of using a commercially available activity tracker to evaluate sleep in the ICU. We evaluated more patients than any prior healthy patient study. ${ }^{12-14}$ Wearable technology such as research-grade actigraphy or other advanced activity watches show potential for various healthcare applications. ${ }^{25}$ The Fitbit Charge 2 was chosen due to its potential for more broad application as it offers the advantage of widespread availability, low cost, favourable patient perception, ease of cleaning and potential for utilisation for other areas than sleep such as mobility (step) tracking both during and after the ICU stay. ${ }^{20}$

When applied to a highly selected group of nonintubated adults, the Fitbit Charge 2 was able to characterise TST for all patients on all ICU nights. However, it was able to characterise awakenings on all ICU nights in only half the patients and sleep stage data on all ICU nights in less than a fifth of the patients. The reason for the high prevalence of missing awakening and sleep stage data remains unclear given TST data were available on all ICU nights and the reported frequency of missing awakening and sleep stage data in healthy adults has been reported to be less than $5 \% .{ }^{12}{ }^{13}$ According to the manufacturer, sleep data are most frequently missing during periods of substantial heart rate variability (HRV), which is not further defined, or when the TST is $<3$ hours. ${ }^{18}$ Of note, the Fitbit Charge 2 relies on detecting the heart rate fluctuation that occurs during sleep stage transitions to differentiate between light, deep and REM sleep. Exact algorithms are considered proprietary by device and not available. Although HRV could be affected by underlying cardiac disease, no patient had sustained nocturnal tachycardia and the TST was $<3$ hours on only $26 \%$ of the nights where awakening and/or sleep stage data were missing.

While the Fitbit did provide data on number of times restless (median (IQR) 7 (3-13)) and the minutes awake (23 (8-41)) for some of the assessments where sleep staging data were unavailable, the relationship between these values and sleep stage tracking is not known. Fragmented sleep, while not evaluated in our study, is common in the ICU and may influence tracker device output. ${ }^{131926}$ The ICU day the assessment was conducted may have also confounded the results we report as the degree of sleepiness during an ICU stay may grow over the course of the admission. Future investigation is required to better understand how patient, time in ICU and sleep-related characteristics differ between critically ill and healthy adults and how these factors affect sleeprelated tracker values.

The TST-RCSQ agreement we report is consistent with Fitbit-generated TST evaluations of healthy adults where TST also correlated moderately with patient-perceived sleep quality. ${ }^{11}$ In other healthy patient studies, Fitbitderived TST has been shown to correlate strongly with TST measured by actigraphy or PSG. ${ }^{12}{ }^{13}$ The Fitbit Charge 2 has been shown to underestimate sleep stage readings with missed \#AW in health populations, ${ }^{12} 1326$ and utility of stage tracking for everyday consumers has been questioned. ${ }^{27}$ While our results suggest a moderate correlation may exist between Fitbit-derived ICU sleep stage data and patient-perceived sleep quality, sleep stage data were available on too few nights to reach significance and make any firm conclusions on sleep stage-RCSQ agreement. These results suggest the need for more study prior to routine use in ICU patients. Given the Fitbit Charge 2 relies on detecting light, deep and REM sleep transitions using a combination of triaxial accelerometry (ie, movement) and optical heart rate sensors, ${ }^{12}$ future studies should also incorporate both continuous patient video and heart rate monitoring.

The RCSQ was able to be completed in $100 \%$ of attempts, a completion rate higher than other studies, ${ }^{28-30}$ that demonstrates its feasibility when administered as a repeated assessment in an ICU cohort. Overall, sleep was classified as fair with a mean RCSQ of $52 \pm 25$, which is comparable to previously reported ICU means of $34-60 .^{28-30}$ The even distribution of RCSQ scores across poor, fair and good sleep groups suggests that all correlation efforts were conducted across the entire spectrum of patient-perceived sleep quality.

Generalisability from our study to all ICU patients is limited given patients with a history of a sleep condition, a factor potentially affecting Fitbit assessment (paraplegia/quadriplegia), or a condition affecting reliable RCSQ completion (eg, delirium) were excluded. While our results cannot be applied to mechanically ventilated adults with a higher severity of illness, it is possible that the Fitbit detection issues we observed in our study may be even greater in sicker ICU populations. The RCSQ has limitations as a method to validate ICU tracker use in the ICU given it cannot reliably estimate TST or \#AW like actigraphy or time spent at each sleep stage like PSG. Moreover, the RCSQ may be subject to potential recall bias and rater fatigue when administered repeatedly. ${ }^{5}$ Although up to $50 \%$ of sleep in critically ill adults occurs during the daytime, we discourage daytime sleeping in our ICUs to facilitate regular daytime mobilisation. ${ }^{19} 31$ Therefore, only nocturnal values were included in our analysis. Future investigations should evaluate the ability of the Fitbit two to detect altered circadian rhythmicity. Based on these concerns and the results of our study, future studies investigating nocturnal sleep tracker use in the ICU should incorporate actigraphy (or PSG), collect heart rate continuously and consider characterising daytime sleep patterns. Newer activity tracker models, not available at the time of our study, incorporate continuous HR measurement in their algorithms and may be options to incorporate into future studies.

\section{Conclusions}

The Fitbit Charge 2 appears to measure TST in nonintubated ICU patients, the results of which correlate moderately with patient-perceived sleep quality. However, 
it cannot consistently measure \#AW or sleep quality in the ICU. Future research is required before commercially available activity trackers should be used to characterise sleep in the ICU.

\section{Author affiliations}

'Department of Pharmacy, AdventHealth Orlando, Orlando, Florida, USA

${ }^{2}$ Department of Pharmacy, Baptist Health, Jacksonville, Florida, USA

${ }^{3}$ Department of Pharmacy, University of Chicago Medical Center, Chicago, Illinois, USA

${ }^{4}$ Pulmonology, Central Florida Pulmonary Group PA, Orlando, Florida, USA

${ }^{5}$ Research Institute, AdventHealth Orlando, Orlando, Florida, USA

${ }^{6}$ School of Pharmacy, Northeastern University, Boston, Massachusetts, USA

${ }^{7}$ Division of Pulmonary, Critical Care and Sleep Medicine, Tufts Medical

Center, Boston, Massachusetts, USA

Contributors PL takes full responsibility for the content of the manuscript. JA, $X T, E P, M A$ and PL were involved in the conception and design of the study and acquired all data. JA, XT, EP, MA, PL,YD and JD analysed the data and drafted the manuscript.

Funding This study was supported by an unrestricted grant from the American Society of Health-System Pharmacists. Neither the sponsor (or the manufacturer of the activity tracker used in the study) had a role in the conception, design or conduct of this study; collection, management, analysis, interpretation or presentation of data; or preparation, review or approval of this manuscript.

Competing interests None declared.

Patient and public involvement Patients and/or the public were not involved in the design, or conduct, or reporting, or dissemination plans of this research.

Patient consent for publication Not required.

Provenance and peer review Not commissioned; externally peer reviewed.

Data availability statement Data are available upon reasonable request.

Open access This is an open access article distributed in accordance with the Creative Commons Attribution Non Commercial (CC BY-NC 4.0) license, which permits others to distribute, remix, adapt, build upon this work non-commercially, and license their derivative works on different terms, provided the original work is properly cited, appropriate credit is given, any changes made indicated, and the use is non-commercial. See: http://creativecommons.org/licenses/by-nc/4.0/.

ORCID iD

Patricia R Louzon http://orcid.org/0000-0001-8824-252X

\section{REFERENCES}

1 Devlin JW, Skrobik Y, Gélinas C, et al. Clinical practice guidelines for the prevention and management of pain, Agitation/Sedation, delirium, immobility, and sleep disruption in adult patients in the ICU. Crit Care Med 2018;46:e825-73.

2 Pisani MA, D'Ambrosio C. Sleep and delirium in adults who are critically ill: a contemporary review. Chest 2020;157:977-84.

3 Knauert MP, Pisani M, Redeker N, et al. Pilot study: an intensive care unit sleep promotion protocol. BMJ Open Respir Res 2019;6:e000411.

4 Kamdar BB, Niessen T, Colantuoni E, et al. Delirium transitions in the medical ICU: exploring the role of sleep quality and other factors. Crit Care Med 2015;43:135-41.

5 Richards KC, O'Sullivan PS, Phillips RL. Measurement of sleep in critically ill patients. J Nurs Meas 2000;8:131-44.

6 Bourne RS, Mills GH. Sleep disruption in critically ill patients-pharmacological considerations. Anaesthesia 2004;59:374-84.

7 Cooper AB, Thornley KS, Young GB, et al. Sleep in critically ill patients requiring mechanical ventilation. Chest 2000;117:809-18.

8 Schwab KE, Ronish B, Needham DM, et al. Actigraphy to evaluate sleep in the intensive care unit. A systematic review. Ann Am Thorac Soc 2018:15:1075-82.
9 Kamdar BB, Kadden DJ, Vangala S, et al. Feasibility of continuous actigraphy in patients in a medical intensive care unit. Am J Crit Care 2017:26:329-35.

10 Lee H-A, Lee H-J, Moon J-H, et al. Comparison of wearable activity tracker with actigraphy for sleep evaluation and circadian restactivity rhythm measurement in healthy young adults. Psychiatry Investig 2017;14:179-85.

11 Lee J-M, Byun W, Keill A, et al. Comparison of Wearable Trackers' Ability to Estimate Sleep. Int J Environ Res Public Health 2018;15:1265.

12 de Zambotti M, Goldstone A, Claudatos S, et al. A validation study of Fitbit charge $2^{\mathrm{TM}}$ compared with polysomnography in adults. Chronobiol Int 2018;35:465-76.

13 de Zambotti M, Baker FC, Willoughby AR, et al. Measures of sleep and cardiac functioning during sleep using a multi-sensory commercially-available wristband in adolescents. Physiol Behav 2016;158:143-9.

14 Evenson KR, Goto MM, Furberg RD, et al. Systematic review of the validity and reliability of consumer-wearable activity trackers. Int $J$ Behav Nutr Phys Act 2015;12:159.

15 Johns MW. A new method for measuring daytime sleepiness: the Epworth Sleepiness scale. Sleep 1991;14:540-5.

16 Freedman NS, Kotzer N, Schwab RJ. Patient perception of sleep quality and etiology of sleep disruption in the intensive care unit. $A m$ $J$ Respir Crit Care Med 1999;159:1155-62.

17 Little A, Ethier C, Ayas N, et al. A patient survey of sleep quality in the intensive care unit. Minerva Anestesiol 2012;78:406-14.

18 Fitbit Customer Service. How do I track my sleep with my Fitbit device?. San Francisco, CA: Fitbit, 2020. https://help.fitbit.com/ articles/en_US/Help_article/2163\#ClassicSleep

19 Elliott R, McKinley S, Cistulli P, et al. Characterisation of sleep in intensive care using 24-hour polysomnography: an observational study. Crit Care 2013;17:R46.

20 Statista. Fitbit's fitness tracker shipment share worldwide from 2013-2016. New York, NY: Statista, 2016. https://www.statista.com/ statistics/795548/fitbit-fitness-trackers-shipment-share-worldwide/

21 Owen, Malcom. Apple watch still dominating the North American wearables market, 2019. Available: https://appleinsider.com/ articles/19/08/15/apple-watch-still-dominating-the-north-americanwearables-market

22 Nicolás A, Aizpitarte E, Iruarrizaga A, et al. Perception of night-time sleep by surgical patients in an intensive care unit. Nurs Crit Care 2008;13:25-33.

23 Hamlett A, Ryan L, Wolfinger R. On the use of PROC MIXED to Estimate Correlation in the Presence of Repeated Measures. Paper 198-29. 2004 SAS Institute Inc. In: Proceedings of the Twenty-Ninth annual $S A S \circledR$ users group International Conference. Cary, NC: SAS Institute Inc, 2004

24 Schober P, Boer C, Schwarte LA. Correlation coefficients: appropriate use and interpretation. Anesth Analg 2018;126:1763-8.

25 Chokshi NP, Adusumalli S, Small DS, et al. Loss-Framed financial incentives and personalized Goal-Setting to increase physical activity among ischemic heart disease patients using wearable devices: the active reward randomized trial. J Am Heart Assoc 2018:7:e009173.

26 Liang Z, Chapa-Martell MA. Accuracy of Fitbit wristbands in measuring sleep stage transitions and the effect of user-specific factors. JMIR Mhealth Uhealth 2019;7:e13384.

27 Zraick K, Mervosh S. That sleep tracker could make your insomnia worse. The New York Times, 2019.

28 Menear A, Elliott R, M Aitken L, et al. Repeated sleep-quality assessment and use of sleep-promoting interventions in ICU. Nurs Crit Care 2017;22:348-54.

29 Kamdar BB, Shah PA, King LM, et al. Patient-nurse interrater reliability and agreement of the Richards-Campbell sleep questionnaire. Am J Crit Care 2012;21:261-9.

30 Alsulami G, Rice AM, Kidd L. Prospective repeated assessment of self-reported sleep quality and sleep disruptive factors in the intensive care unit: acceptability of daily assessment of sleep quality. BMJ Open 2019;9:e029957.

31 Friese RS, Diaz-Arrastia R, McBride D, et al. Quantity and quality of sleep in the surgical intensive care unit: are our patients sleeping? $J$ Trauma 2007;63:1210-4. 